\title{
ON THE VALUE OF OPTIONS IN CERTAIN CONTRACTS.
}

\author{
To the Editor.
}

SIR,-The equation of condition at the end of my letter in the last Number of the Journal needs a correction, owing to the accidental omission of a factor. The equation should be

$$
\mathrm{Q} \cdot \mathrm{P}_{x}+\frac{\lambda_{n}}{l_{x+n}} \cdot \frac{\mathrm{N}_{x+n}}{\mathrm{D}_{x}}=\mathrm{P}_{x}
$$

The annuity portion of the risk is evidently $=\frac{\lambda_{n}}{l_{x}} v^{n} \cdot a_{x+n}$, or $\frac{\lambda_{n}}{l_{x+n}} \cdot \frac{N_{x+n}}{D_{x}}$, since $\frac{\lambda_{n}}{l_{x}}$ is the probability that any one of the lives will enter upon the annuity. Hence

$$
P_{x}=\frac{\lambda_{n}}{l_{x+n}} \cdot \frac{\mathrm{N}_{x+n}}{(1-Q) \mathrm{D}_{x}}
$$

Taking the example given in my letter, we have, as before, $Q=422556$ and $\lambda_{10}=2170 \cdot 32$; hence $P_{x}=5 \cdot 6094$.

To determine the precise effect of the withdrawals upon the amonnt of the preminm, we must find what the preminm would be if it were returnable at the moment of death and no withdrawals were allowed. In this case it is easily found that $P_{x}=\frac{\mathrm{N}_{x+n}}{\mathrm{D}_{x}-\frac{i}{\log (1+i)}\left(\mathrm{M}_{x}-\mathrm{M}_{x+n}\right)}=6.2828$.

It therefore appears that if the withdrawals which have been assumed were certain to take place, the effect wonld be to lessen the preminm, not to increase it.

I did not perceive the omission of the factor referred to until the day the Journal was published, otherwise the numerical example before given would have been corrected, and the paradoxical nature of its result wonld thus have been removed.

At whatever time a withdrawal takes place, it is obvious that the return of the original premium without interest must leave the Office a gainer, because the risk continually increases, while the preminm returned merely represents the initial value of the risk. Thus the Office is set free from a liability without being called upon to pay its fnll equivalent valne; hence the reduction which takes place in the premium.

I am, Sir,

$$
\text { Your obedient servant, }
$$

